
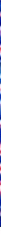

Research Article

\title{
Experimental neutron pole figures of minerals composing the bivalve mollusc shells
}

\author{
Dmitry Nikolayev $^{1}$ (D) $\cdot$ Tatiana Lychagina $^{1} \cdot$ Alexey Pakhnevich $^{2}$
}

(c) Springer Nature Switzerland AG 2019

\begin{abstract}
Recently, several studies have focused on the crystallographic texture of bivalve mollusc shells. Unfortunately, these investigations have been limited to the local level. We demonstrate the similarities and differences between the texture measured over the whole shell and that measured over a small part of a shell. An analysis of the global crystallographic texture of bivalve mollusc shells of different genera was carried out using time-of-flight neutron diffraction. The reason for this analysis was to determine whether the crystallographic texture character was similar within the class Bivalvia. It was observed that the shells of mollusc species of the genus Mytilus consist of two phases, calcite and aragonite. Ostrea edulis shells consist of almost pure calcite, whereas Mya arenaria shells consist of almost pure aragonite. It was concluded that the character of the global textures of the different phases in the same shells is different. The advantages of characterisation of the global crystallographic texture are also discussed.
\end{abstract}

Keywords Crystallographic texture · Neutron diffraction · Bivalve mollusc shells · Pole figures

\section{Introduction}

Driven by the need for new lightweight composites with improved mechanical properties, and cost-effective and environmentally friendly manufacturing strategies [1, 2], many engineers are now turning to nature for design insights into the fabrication of damage-tolerant and hierarchically ordered structural materials [3-5]. Biomineralised composites such as bones, sponge spicules and mollusc shells demonstrate excellent mechanical properties, especially considering their relatively soft/weak organic and stiff/brittle ceramic constituents [6-10].

A study of the mineral composition of Bivalvia mollusc shells and the orientational distribution of crystals in these shells may be useful in solving a number of fundamental problems. Previous attempts have been made to compare the mineral composition and texture of the shell crystals of Bivalvia molluscs from different families [11]. It should be noted that throughout this manuscript, we use the term 'texture' as synonym for the term 'crystallographic texture', i.e. the preferred orientation of crystallites. The neutron diffraction method can give the most complete information about the mineral composition and texture features of the crystals.

Biologists and palaeontologists are interested not only in the mineral composition of these shells but also its changes over thousands and millions of years. It is known that a shell falling into the bottom sediment loses organic matter and can be recrystallised, with this matter being replaced by other minerals. This process can be traced by examining subfossil and fossil shells of different geological ages. It is also important in an understanding of the fossilisation processes in mineral skeletons, which is still poorly understood. The rate of change in the texture of crystals without an organic matrix is also interesting.

$\triangle$ Dmitry Nikolayev, dmitry@nf.jinr.ru; Tatiana Lychagina, lychagina@jinr.ru; Alexey Pakhnevich, alvpb@mail.ru | ${ }^{1}$ Frank Laboratory of Neutron Physics, Joint Institute for Nuclear Research, Dubna, Russia. ${ }^{2}$ Borissiak Paleontological Institute, Russian Academy of Sciences, Moscow, Russia. 
Several recent studies have focused on the crystallographic texture of biological objects. It has been found that polycrystalline physical or mechanical properties very often exhibit an anisotropy, which is mainly due to the presence of preferred orientations or crystallographic texture, as described by an orientation distribution function (ODF). Both aspects, that is, the characterisation of the texture and the correlation of this with the properties, are essential in understanding the behaviour of the polycrystalline biological materials. A quantitative description of the crystallographic texture relies on a statistically representative set of the crystalline orientations, and information about the ODF is typically extracted from experimentally measured pole figures. The pole figure function $P_{h}(y)$ gives the volume fraction of the sample for which the lattice plane normal $h$ falls in various sample directions $y$. It should be noted that electron backscatter diffraction (EBSD), which is a very widespread and acknowledged method, does not rely on pole figure measurements. ODF can be directly measured by this method, and it should be emphasised that the pole figure characterises a sample rather than whole object from which the sample was extracted.

Crystallographic texture is the set of crystalline orientations in a polycrystalline material and can be used to fingerprint the 'history' of the material. In addition, it carries information about inelastic deformation regimes (related to dislocation glide, twinning, etc.), crystallisation/recrystallisation, and sometimes structural phase transitions or sedimentation. Crystallographic texture also has a direct relationship with the material properties. There are three scales of experimental information about crystallographic texture: EBSD or scanning electron microscopy (SEM); $X$-rays; and thermal neutron diffraction. EBSD and SEM deal only with the surface of a material, with a penetration depth of about $0.5-1 \mu \mathrm{m}$. A local analysis of shell texture carried out with the help of $X$-rays can provide information from a small surface. The penetrating ability of the X-ray method is on the order of tens of microns and is insufficient to interpret the general features of a shell's texture. The mollusc shells consist of several layers with differently oriented elements of the microstructure (fibres, prisms, etc.), and it is impossible to take into account the crystal orientation of the elements in all of these layers using the $\mathrm{X}$-ray method. Moreover, in some species of bivalve molluscs, the mineral composition may differ between layers; for example, aragonite or calcite may predominate. The number of crystals that can be analysed by the neutron method is much larger than by local X-ray analysis, since the penetration depth of neutrons is measured in centimetres. Thus, the real global texture can be measured by means of neutron diffraction. The local texture characterises only one small part of an object, whereas the global texture enables us to draw conclusions about an object as a whole.

The EBSD technique has been used to obtain crystallographic texture information about bivalve mollusc shells [11-13], and the pole figures for these shells have been measured with X-rays [14]. We use neutron time-offlight diffraction to measure pole figures. In case of neutron pole figures, the sample is the investigated object itself. It is also worth mentioning that the X-ray and EBSD techniques have special requirements, in that a sample must be flat, whereas a sample for neutron measurements does not require special preparation; the mollusc shells can be measured as they are, by stacking several of them to increase the sample volume and statistical representativity.

In this study, we explore the global crystallographic texture of bivalve molluscs. The mollusc species of genus Mytilus is very widespread in modern seas and sedimentary rocks. Mytilus is a cosmopolitan genus of medium-tolarge, edible saltwater mussels; these are bivalve molluscs in the family Mytilidae. Mytilus shells possess both calcite and aragonite phases with different content. The local crystallographic texture of Mytilus shells was studied in [11-13], and the global texture of Mya arenaria (Linnaeus, 1758) and Ostrea edulis (Linnaeus, 1758) has also been investigated.

We carry out a comparative analysis of the global crystallographic texture of the above-mentioned bivalve mollusc shells for the two phases of calcite and aragonite. The primary reason for this analysis is to determine whether the crystallographic texture character is similar across the class Bivalvia.

We also examine whether the sharpness of the crystallographic texture for the calcite and aragonite phases is of the same order. The question of the textural sharpness of the different phases in the same sample of a natural species is important.

\section{Materials and methods}

\subsection{Time-of-flight neutron texture measurements}

Measurements of the crystallographic texture were carried out with a SKAT spectrometer, situated at beam 7A of the IBR-2 pulsed nuclear reactor [15, 16] (JINR, Dubna, Russia). The SKAT spectrometer has 19 detectors arranged at the same angle of $90^{\circ}$ to the neutron beam, allowing us to obtain patterns under the same conditions. The long flight path of more than $100 \mathrm{~m}$ and the collimator system provide a relatively high resolution of $\Delta d / d=5 \times 10^{-3}$ at $d=2.5 \AA$. A sample is rotated in the goniometer through $360^{\circ}$ about the horizontal axis, with a step of $5^{\circ}$, and 
$19 \times 72=1368$ partial patterns are recorded for the same number of crystal orientations. The cross section of the beam $(50 \times 90 \mathrm{~mm})$ enables distinct diffraction from sample volumes of up to $100 \mathrm{~cm}^{3}$. The typical shell size is a few millimetres less than $50 \mathrm{~mm}$, meaning that the whole shell will fit into the beam cross section during the rotation in the goniometer, as shown in Fig. 1. Figure 2 shows an example of the resulting pattern obtained for the Mytilus shell sample, based on the summation of 1368 individual patterns.

Complete pole figures were extracted from the measured patterns, and integrated intensities were placed to stereographic projection and normalised. The local peak fit was used to accomplish pole figure extraction [17], and particular attention was paid to analysis of

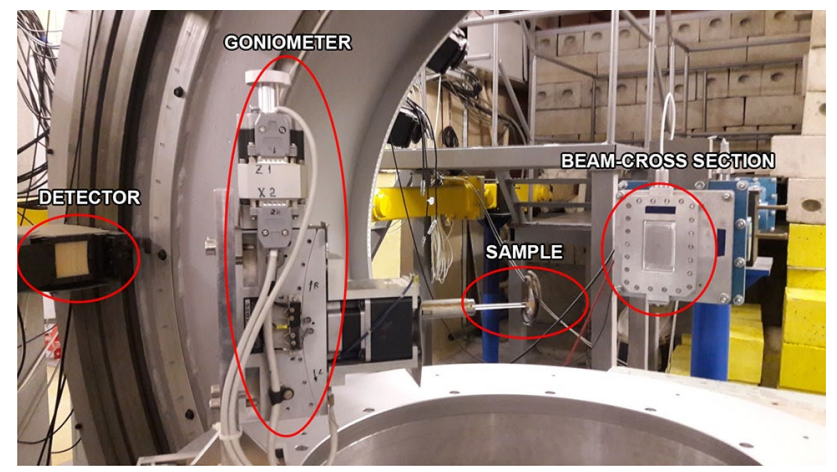

the pole figures [18]. It should be mentioned that each individual pattern was measured under the same conditions, meaning that the pole figures obtained in this way do not need correction. A further advantage of neutron diffraction experiments is that the flexibility in the sample geometry is suitable for neutron texture analysis so that in most cases only minimal sample preparation is required. Due to the large penetration depth of neutrons, bulk samples of centimetre size can readily be investigated in transmission geometry.

\subsection{Sample collection}

Shells of Mytilus galloprovincialis, Mya arenaria and Ostrea edulis were gathered from coastal emissions near Pesotchnoe village in Kazantip Bay, on the coast of the Azov sea, in the summers of 2009-2013. Shells of Mytilus edulis were collected from the North Sea coast (littoral emission) at Oostende, Belgium, in May 2014. Mytilus trossulus shells were collected in 1999 from Adak island (Aleutian islands) on a littoral. This collection was stored in the Laboratory for Historical Ecology of the Severtsov Institute of Ecology and Evolution, Russian Academy of Sciences, Moscow, Russia. All other collections were made by researchers from the Borissiak Paleontological Institute, Russian Academy of Sciences, Moscow, Russia.

Fig. 1 Position of the sample in the SKAT diffractometer

Fig. 2 Neutron diffraction pattern for Mytilus edulis shells

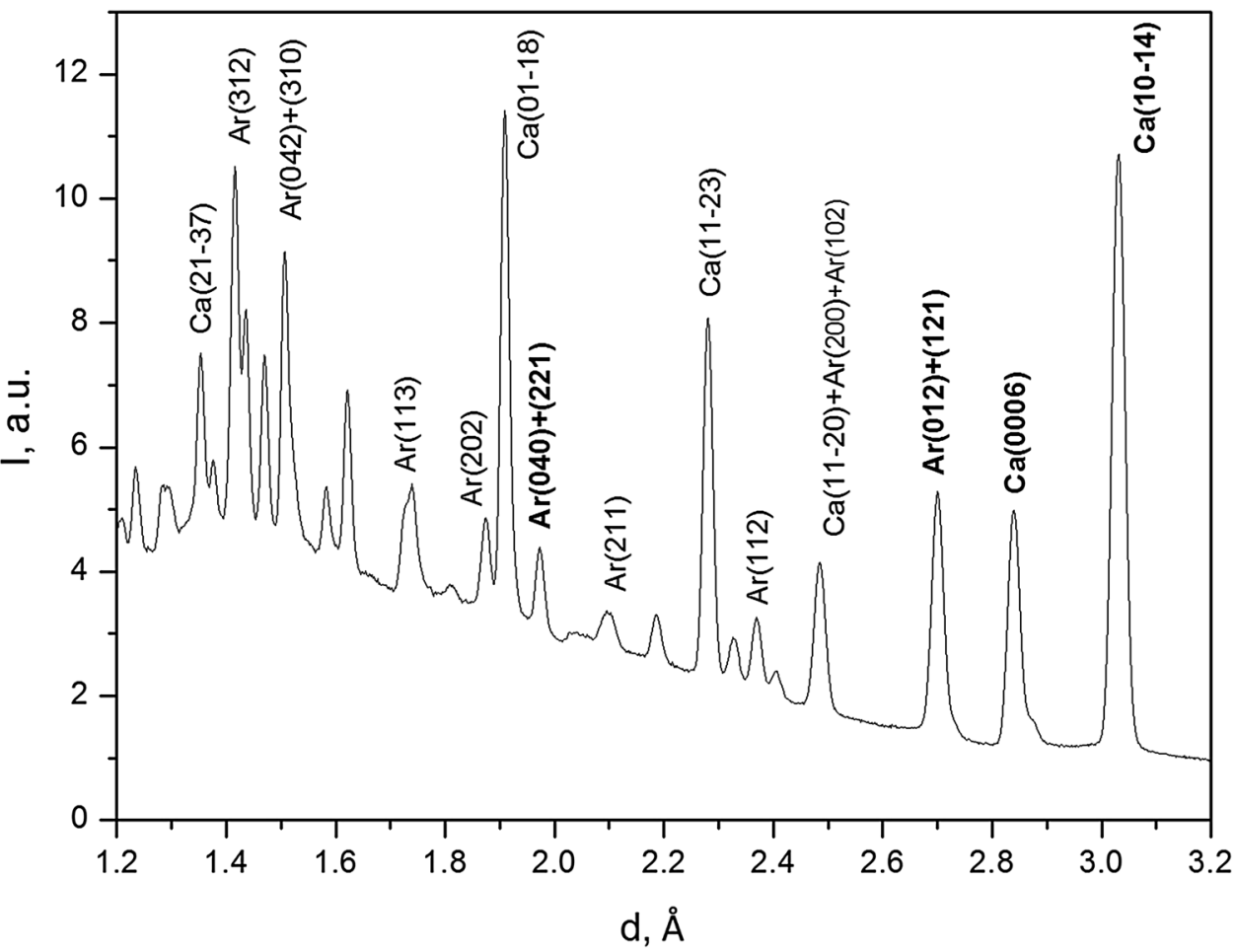

SN Applied Sciences A SPRINGER NATURE journa 


\section{Results}

We studied shells of Mytilus galloprovincialis (Lamarck, 1819) from the Sea of Azov; Mytilus edulis (Linnaeus, 1758) from the North Sea; and Mytilus trossulus (Gould, 1850) from the coast of the Aleutian islands. All samples were recently collected ashore. The measured neutron diffraction pattern is shown in Fig. 2. All Mytilus shells possessed both calcite and aragonite phases.

Mya arenaria shell had only an aragonite phase, whereas Ostrea edulis had only a calcite phase. Figure 3 displays the sum neutron diffraction patterns measured for these shells.

Pole figures for the calcite and aragonite phases for different Mytilus shells are shown in Fig. 4. It can be seen that the calcite pole figures (0006) are qualitatively very similar for different species of Mytilus genus, and are very sharp. The pole figure maximum for the Mytilus galloprovincialis sample is $11.61 \mathrm{mrd}$, whereas for the Mytilus trossulus sample is $13.27 \mathrm{mrd}$, and for Mytilus edulis, 13.15 $\mathrm{mrd}$. A commonly used convention is to express the data in multiples of a random orientation distribution (mrd), meaning that the pole figure of a random uniform standard sample has an intensity of one at all points. Pole figure regions with intensities higher than this indicate that more lattice planes are aligned in those directions than in a sample with a random texture. A number of results have been reported [19-21] of measured calcite pole figures for limestone and marble, and in all of these, the maximum values of the pole figures are no higher than $7 \mathrm{mrd}$. However, in [22], it was shown that only very strong torsional deformation of marble samples at high temperatures can lead to a sharp texture with a maximum value for the pole figures of higher than $8 \mathrm{mrd}$. The crystallographic texture of the Mytilus calcite phase is very sharp compared with that of rocks (marble, limestone), and this means that in biological objects, the texture is about twice as sharp as in non-biological samples. Although there are rocks that are formed from almost pure calcite, rocks consisting of pure aragonite are very rare.

Pole figures $(012)+(121)$ for aragonite phase are also shown in Fig. 4. It can be seen that these pole figures are qualitatively similar but not very sharp. The pole figure maximum for the Mytilus galloprovincialis sample is 3.21 mrd; for Mytilus trossulus, this is $3.45 \mathrm{mrd}$; and for Mytilus edulis, $3.36 \mathrm{mrd}$. It is also clear that the shape of the maxima for the aragonite phase is connected with the shape of the shells.

Pole figures for the calcite phase for Ostrea edulis are presented in Fig. 5. As shown in Fig. 3, Ostrea edulis consists only of calcite. The crystallographic texture of Ostrea edulis has a different appearance from that of the calcite phase of Mytilus edulis, and is not sharp. It has a maximum value of $2.46 \mathrm{mrd}$ for the (0006) pole figure and $2.38 \mathrm{mrd}$ for the (10-14) figure, but the shape of the maxima is not similar to the shape of the shell.
Fig. 3 Neutron diffraction patterns measured for Mya arenaria (sand shell) and Ostrea edulis

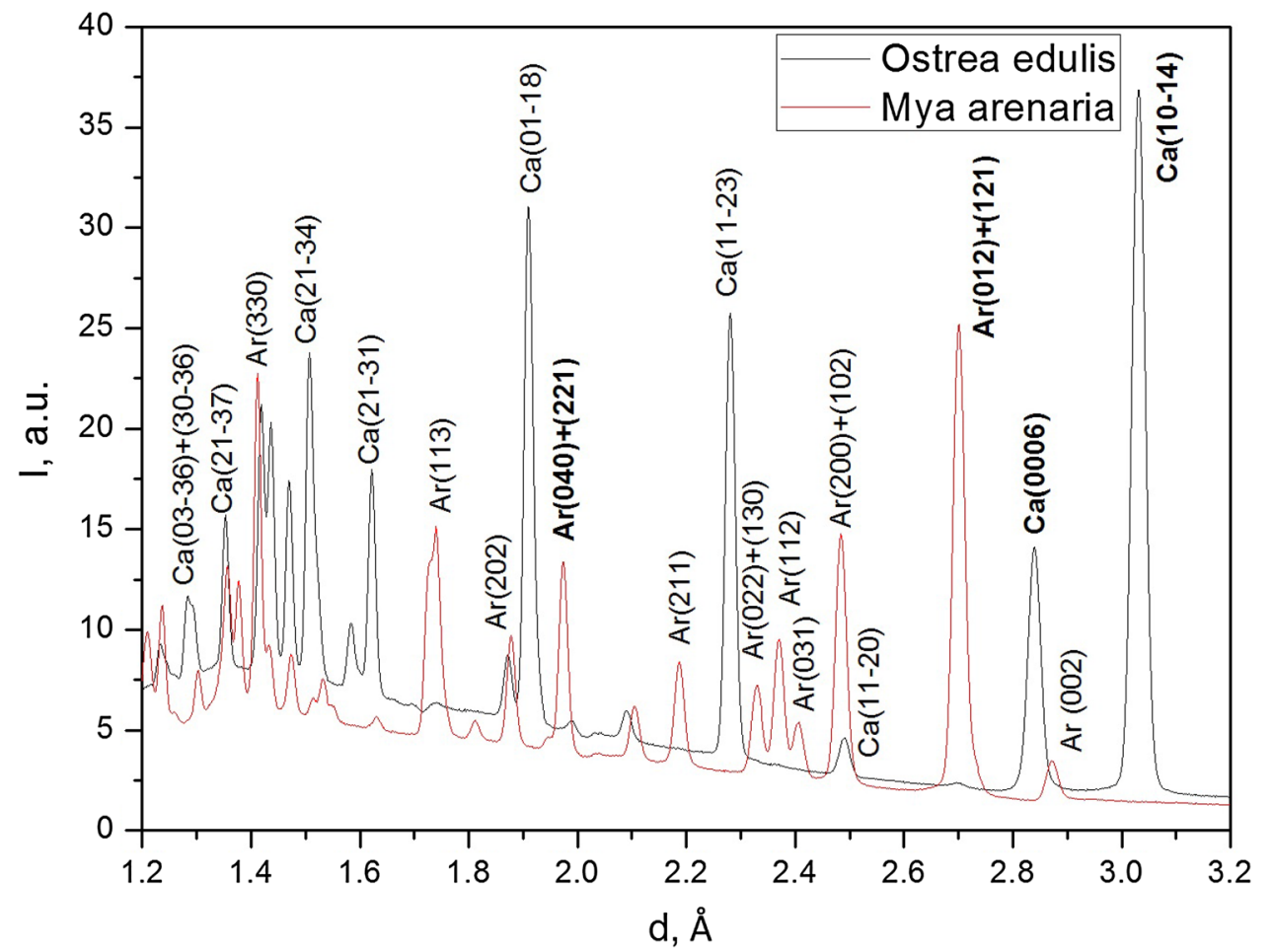


Fig. 4 Pole figures for the calcite and aragonite phases of Mytilus shells; the colour scale in the top right-hand corner indicates the intensity of isolines, shown in units of random distribution (mrd)

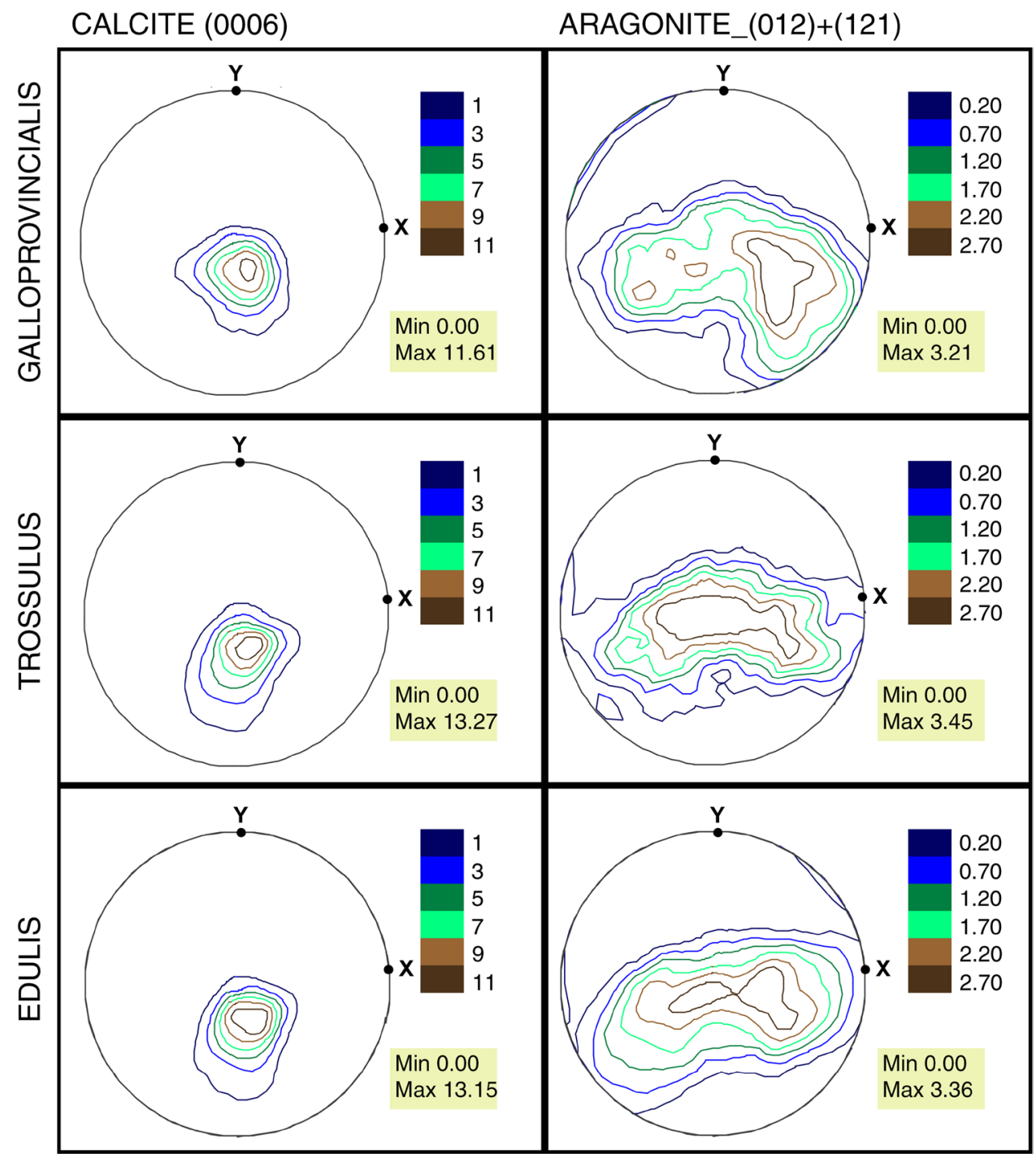

The $(012)+(121)$ and $(040)+(221)$ pole figures for the aragonite phase of Mya arenaria are shown in Fig. 6. A comparison with the crystallographic texture of Mytilus edulis is also presented in this figure. As shown in Fig. 3, Mya arenaria consists only of aragonite. It has a maximum value of $2.63 \mathrm{mrd}$ for the $(012)+(121)$ pole figure, and 1.81 mrd for the $(040)+(221)$ pole figure. The shape of the maxima is connected with the shape of the shell.

In the next section, we discuss the differences between the global crystallographic texture for the bivalve molluscs and local textures reported by other researchers.

\section{Discussion}

We have shown that Mytilus shells consist of two global phases, calcite and aragonite, and that the calcite phase has a very sharp texture, in contrast the relatively weak texture of aragonite. The crystallographic texture of corresponding phases for samples of different Mytilus species is qualitatively similar, and the difference appears at a quantitative level. The difference between the pole figure maxima for Mytilus galloprovincialis and Mytilus trossulus is approximately $12.5 \%$.

The sample examined here is shown in Fig. 7. To increase the volume of the sample, several shells were glued together, and a glass sample holder was also glued to the above-mentioned shell set. The green arrow shown on the sample holder indicates the centre of a pole figure. The calcite trigonal crystallites are shown by a prism with direction (0006), as indicated by the red arrow. The position of the maxima for PF (0006) of the calcite phase of Mytilus shells (Fig. 4) means that most calcite crystallites are oriented in such a way that there is a difference of $20^{\circ}-25^{\circ}$ between the directions marked by the red and green arrows. 
Fig. 5 Pole figures for the calcite phase of Ostrea edulis and Mytilus edulis shells; the colour scale in the top right-hand corner indicates the intensity of isolines, presented in units of random distribution (mrd)

Fig. 6 Pole figures for the aragonite phase of Mya arenaria and Mytilus edulis shells; the colour scale in the top right-hand corner indicates the intensity of isolines, shown in units of random distribution (mrd)
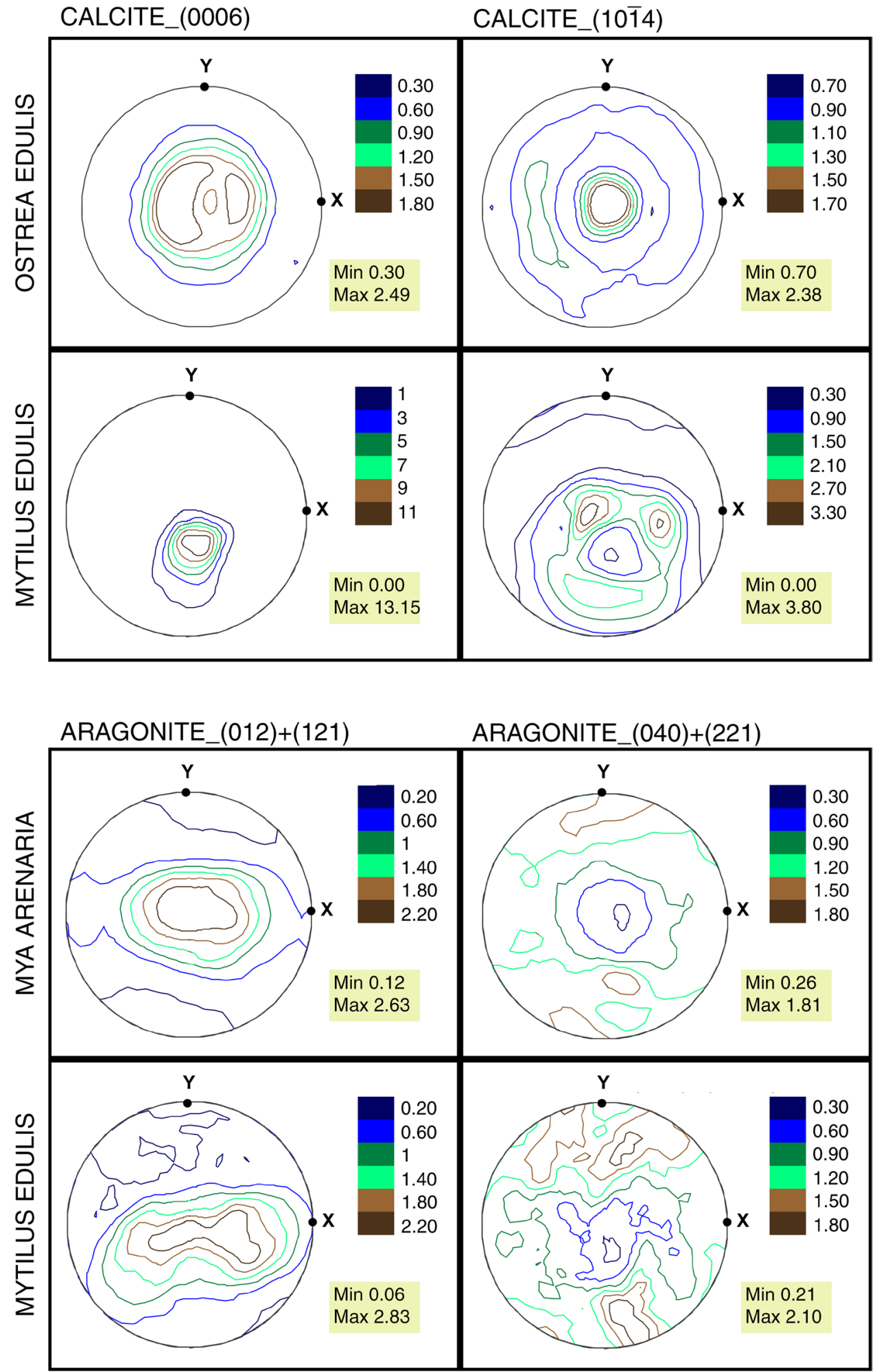

Figure 7 displays the neutron measurements coordinate system connected with the object. For the $\mathrm{X}$-ray measurements, a sample is a flat piece of shell, with a coordinate system aligned with the specific piece. It is clear that due to the shape of the shell, the orientation of the coordinate axis changes between samples, even for the same object, inevitably leading to an ambiguous texture description of the overall shell.

The volume fraction of shell regions misoriented with respect to the goniometer axes can influence the 
Fig. 7 An explanation for the maxima in PF (0006) for the calcite phase of Mytilus shells. The calcite unit lattice is shown in black in the right-hand insert. The crystallographic indices of the calcite planes are connected with the Bravais lattice, shown in grey. The triangular prism is shown to the left, marked in purple

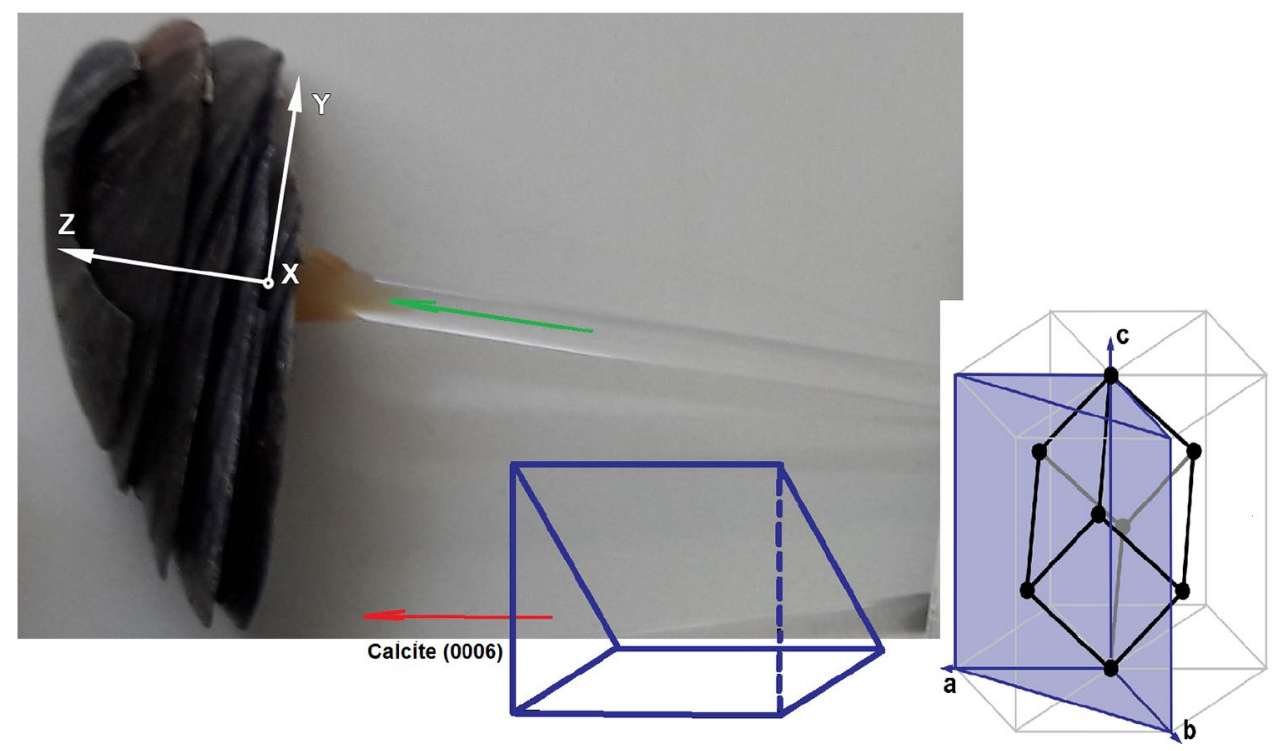

symmetry and shape of the textures. If the dependence of these textures on the sample axes is large, dependence on the shell shape may arise; if it is low or absent, the global texture may be less influenced by the fact that we are averaging with respect to different sample axes. A more detailed discussion of global and local texture is presented in $[23,24]$.

The local crystallographic texture of nacre (aragonite) was measured for eleven bivalve species of three different superorders (see [11]). The pole figures for Mytilus galloprovincialis and Mytilus edulis are also presented. All pole figures were obtained using the EBSD experimental technique. The typical area analysed was much less than one square millimetre. On the basis of these measurements, authors characterised the crystallographic texture of the whole shell, an approach that implicitly assumes that the texture is homogenous over the whole shell. The global texture of the aragonite phase presented in Fig. 4 is somewhat qualitatively similar to the results in [11], but the global texture shows a dependence on the shell shape for all shells containing aragonite.

The local crystallographic texture of calcite was measured for Mytilus galloprovincialis (see [12]). All pole figures presented here were obtained using the EBSD experimental technique. The pole figures shown in Fig. 6 in [12] were measured for a different part of a shell and have a different orientation of the main maximum; the authors explained this phenomenon by the influence of the $\mathrm{pH}$ gradient of the environment in which the mussels grew. Texture measurements were carried out on a shell area of less than one square millimetre, and the local texture can change significantly even within one shell. To characterise these local changes (for example, those connected with environmental changes), a knowledge of the local texture is required. Global texture measurements are preferable to characterise the whole shell.

The global pole figures for the calcite phase of Mytilus galloprovincialis were measured by neutron diffraction and are presented in Fig. 4; these also have a textural component that is similar to that of the local calcite pole figures, but it is quantitatively less sharp than reported in [12].

\section{Conclusion}

On the basis of the results presented here, we can conclude the following. All Mytilus shell samples consist of both calcite and aragonite phases; the Ostrea edulis shell sample is formed from almost pure calcite; and Mya arenaria is formed from almost pure aragonite. The crystallographic texture of the Mytilus calcite phase is very sharp, whereas that of the aragonite phase is not. The crystallographic textures of the samples of the Mytilus genus studied here are qualitatively very similar. We observe that the character of the crystallographic texture of the samples from the same (bivalve) class but of a different genus (Mya, Ostrea) exhibits differences from that of the Mytilus genus.

We can conclude that the crystallographic texture of the calcite phase is not connected with the shell shape, unlike that of the aragonite phase, for the samples studied here. The question of the correlation between the textures of aragonite and calcite is very important; however, it is likely that we have insufficient experimental results to judge this correlation reliably.

The global crystallographic texture is both qualitatively similar and quantitatively different from the local texture. It enables us to characterise the whole object, since the local texture may be different for different parts of the shell. The 
neutron analysis of mussel shells showed a general overall tendency for the texture of all calcite crystals, which could not be obtained by the X-ray or EBSD methods.

Acknowledgements This work was supported by the Joint Institute for Nuclear Research (04-4-1121-2015/2020; RO-JINR Projects Nos. 219/10.04.2017 and 220/10.04.2017 item 43; RO-JINR Projects Nos. $323 / 21.05 .2018$ and 322/21.05.2018 items 35, 62).

\section{Compliance with ethical standards}

Conflict of interest The authors declare that they have no conflict of interest.

\section{References}

1. Munch E, Launey ME, Alsem DH, Saiz E, Tomsia AP, Ritchie RO (2008) Tough, bio-inspired hybrid materials. Science 322:1516-1520

2. Erb RM, Libanori R, Rothfuchs N, Studart AR (2011) Composites reinforced in three dimensions by using low magnetic fields. Science 335:199-204

3. Ortiz C, Boyce MC (2008) Bioinspired structural materials. Science 319:1053-1054

4. Dunlop JWC, Fratzl P (2010) Biological composites. Annu Rev Mater Res 40:1-24

5. Studart AR (2012) Towards high-performance bioinspired composites. Adv Mater 24:5024-5044

6. Tai K, Dao M, Suresh S, Palazoglu A, Ortiz C (2007) Nanoscale heterogeneity promotes energy dissipation in bone. Nat Mater 6:454-462

7. Kamat S, Su X, Ballarini R, Heuer AH (2000) Structural basis for the fracture toughness of the shell of the conch Strombus gigas. Nature 405:1036-1040

8. Aizenberg J, Weaver JC, Thanawala MS, Sundar VC, Morse DE, Fratzl P (2005) Skeleton of Euplectella sp.: structural hierarchy from the nanoscale to the macroscale. Science 309:275-278

9. Li L, Ortiz C (2013) Biological design for simultaneous optical transparency and mechanical robustness in the shell of Placuna placenta. Adv Mater 25:2344-2350

10. Li L, Ortiz C (2014) Pervasive nanoscale deformation twinning as a catalyst for efficient energy dissipation in a bioceramic armour. Nat Mater 13:501-507

11. Fryda J, Klicnarova K, Frydova B, Mergl M (2010) Variability in the crystallographic texture of bivalve nacre. Bull Geosci 85(4):645-662

12. Hahn S, Rodolfo-Metalpa R, Griesshaber E, Schmahl WW, Buhl D, Hall-Spencer JM, Baggini C, Fehr KT, Immenhauser A (2012)
Marine bivalve shell geochemistry and ultrastructure from modern low pH environments: environmental effect versus experimental bias. Biogeosciences 9:1897-1914

13. Fitzer SC, Phoenix VR, Cusack M, Kamenos NA (2014) Ocean acidification impacts mussel control on biomineralisation. Sci Rep. https://doi.org/10.1038/srep06218

14. Hedegaard C, Wenk H-R (1998) Microstructure and texture patterns of mollusc shells. J Molluscan Stud 64:133-136

15. Ullemeyer K, Spalthoff P, Heinitz J, Isakov NN, Nikitin AN, Weber $K$ (1998) The SKAT texture diffractometer at the pulsed reactor IBR-2 at Dubna: experimental layout and first measurements. Nucl Instrum Methods Phys Res 412(1):80-88

16. Keppler R, Ullemeyer K, Behrmann JH, Stipp M (2014) Potential of full pattern fit methods for the texture analysis of geological materials: implications from texture measurements at the recently upgraded neutron time-of-flight diffractometer SKAT. J Appl Cryst 47:1520-1535

17. Lychagina TA, Nikolaev DI (2007) Investigation of experimental pole-figure errors by simulation of individual spectra. Crystallogr Rep 52:774-780

18. Lychagina TA, Nikolayev DI (2016) Quantitative comparison of measured crystallographic texture. J Appl Cryst 49:1290-1299

19. Koch A, Siegesmund S (2004) The combined effect of moisture and temperature on the anomalous behaviour of marbles. Environ Geol 46(3-4):350-363

20. Scheffzuk C, Siegesmund S, Nikolayev DI, Hoffmann A (2007) Texture, spatial and orientation dependence of internal strains in marble: a key to understanding the bowing of marble panels. In: Prikryl R, Smith BJ (eds) Building stone decay: from diagnosis to conservation. Geological Society, London, Special Publications 271, pp 237-250

21. Burlini L, Marquer D, Challandes N, Mazzola S, Zangarini N (1998) Seismic properties of highly strained marbles from the Spliigenpass, central Alps. J Struct Geol 20(213):277-292

22. Pieri M, Kunze K, Burlini L, Stretton I, Olgaard DL, Burg J-P, Wenk H-R (2001) Texture development of calcite by deformation and dynamic recrystallization at $1000 \mathrm{~K}$ during torsion experiments of marble to large strains. Tectonophysics 330:119-140

23. Brokmeier H-G (1999) Advances and applications of neutron texture analysis. Textures Microstruct 33:13-33

24. Brokmeier H-G (2006) Global crystallographic textures obtained by neutron and synchrotron radiation. Physica $B$ 385-386:623-625

Publisher's Note Springer Nature remains neutral with regard to jurisdictional claims in published maps and institutional affiliations. 